\title{
ASTEROID ALBEDOS AND DIAMETERS
}

\author{
EDWARD F. TEDESCO \\ Mission Research Corporation, One Tara Boulevard, Nashua, NH 03062 USA \\ E-mail etedesco@pldac.plh.af.mil
}

\begin{abstract}
This paper discusses the status of our knowledge regarding asteroid sizes and albedos. It presents a brief discussion of the methods used to determine sizes and albedos beginning with attempts using filar micrometers as early as 1802 and culminating with the 1993 publication of the IRAS Minor Planet Survey. It addresses the completeness and reliability of the IRAS Minor Planet Survey, summarizes the statistical adjustments made to the derived results, and considers the implications of those results.
\end{abstract}

\section{Techniques}

The first attempts to measure diameters of asteroids employed direct measurements of their seeing disks using filar micrometers. The earliest such measurements were made by Herschel in 1802 and Schroeter in 1805 (cf., Barnard, 1894). By 1881 thirteen determinations of the diameters of the four brightest asteroids had been published. The most notable feature of these measurements was their large scatter, e.g., up to a factor of ten in the diameter of Ceres. This led Barnard (1894, p. 342) to write that : "... the previous measures of these bodies are very discordant, and were evidently made with instruments too small to deal with the subject." Thus between 1894 and 1900, using a filar micrometer on the Lick Observatory 36-inch, and Yerkes 40-inch, refractors Barnard undertook to measure the diameters of the four brightest asteroids (Barnard, 1894, 1895, 1900a,b). His results are compared with those from current techniques in the section on the largest asteroids, below. See Dollfus (1971) for a summary of direct visual diameter determinations made through 1969.

Modern techniques used to determine asteroid diameters include polarimetry, radar, speckle imaging, stellar occultations, and thermal radiometry. Soon imaging at optical and near-infrared wavelengths, using the Hubble Space Telescope and large ground-based telescopes equipped with adaptive optics systems, will permit direct diameter determinations for many of the larger asteroids. Of these, only the radiometric method is capable of providing results for significant numbers of asteroids.

Measurements of the linear polarization of the reflected light from asteroids as a function of phase angle has been used to determine their diameters. The polarimetric method yields the albedo directly, through the empirically-derived so-called "slope-albedo relation" (KenKnight, et al., 1967). To date "polarimetric diameters" have been published for about 30 asteroids ( $c f$. , Morrison and Zellner, 1979). Polarimetric observations to derive diameters have not been used since the late 1970's for two reasons. First, unlike the other methods discussed here which, in principle, require observations from only a single night, the polarimetric method requires measurements from several nights spaced over a number of weeks in order to define the slope of the polarization - phase angle curve. Second, it was found 
that the slope-albedo relation saturates for low-albedo materials (Zellner et al., $1977 \mathrm{a}, \mathrm{b})$. This means that one cannot distinguish among asteroids with albedos less than about 0.1 on the current H, G magnitude system albedo scale. See Dollfus et al. (1989) for a review of the current state of the art in asteroid polarimetry.

Radar observations in which the target is not actually resolved, i.e., those in which one-dimensional images are obtained, require knowledge of the asteroid's spin rate and pole orientation for an accurate diameter determination. In the absence of these parameters, radar observations set a lower bound on the asteroid's dimensions and pole-on projected area. In the ten years following its proposed upgrade the Arecibo radar telescope will be capable of providing two-dimensional images $(\gg 10$ pixels on target) for two dozen main belt asteroids and a similar number of nearEarth objects, plus accurate lower bounds on the dimensions of over 100 objects (Ostro, 1989).

Speckle imaging has been used to derive shapes, pole orientations, and sizes for six asteroids (Drummond and Hege, 1989). Due to the relatively bright limiting magnitude of this technique its future application is probably limited to a few score asteroids.

Stellar occultations, the passage of an asteroid in front of a star, provide the most accurate asteroid diameters. Measurement of the time interval during which the star is occulted gives the length of the chord across the asteroid. Given a number of well-placed chords one can map the apparent limb profile of the asteroid with great accuracy. If asteroids were spherical, a single well-observed occultation would result in an accurate diameter. In fact, however, most asteroids are significantly nonspherical as indicated by their lightcurves. Hence, a single occultation observation can yield a highly precise result which turns out to be of low accuracy.

Occultation observers are well aware of this situation. Where possible, lightcurves contemporaneous with the occultation event are obtained and used to estimate the three-dimensional shape and thus the mean diameter $\equiv(a * b * c)^{\frac{1}{3}}$ and to adjust the uncertainty.

While occultations of stars by asteroids is a common occurrence, observations of these events remain rare - perhaps one or two per year on average. To date accurate diameters based upon occultation data are available for fewer than 20 asteroids. Even at the rate of two additional asteroids per year it will be many decades before this number reaches 100 . Thus, in the context of determining diameters for statistically significant numbers of asteroids, the greatest value in occultation diameters lies in the calibration of the indirect techniques, primarily the polarimetric and radiometric methods. See Millis and Dunham (1989) for a review of the asteroid stellar occultation method.

The radiometric method makes use of the fact that the thermal radiation emitted by an asteroid must equal the solar radiation it absorbs. For an asteroid at a given heliocentric distance the amount of this energy depends upon the asteroid's size and albedo. By measuring the visual (reflected) brightness and the thermal (emitted) flux a size and albedo can be deduced. The accuracy of the derived size and albedo is a function of the assumptions made in the calculation of the emitted thermal energy, i.e., in the adequacy of the "thermal model". An explication of the thermal model is beyond the scope of this paper. See Lebofsky and Spencer (1989) for a 
recent review. The thermal model used to reduce the IRAS asteroid data is that of Lebofsky et al. (1986a,b).

The accuracy of a radiometric diameter is also limited by the infrared flux calibration and knowledge of the visual brightness at the time of the infrared observation and its extrapolation to zero phase angle. (The visual flux at the time of a thermal infrared observation is almost always calculated from the asteroid's absolute magnitude and an assumed phase function.) Finally, flux variations due to rotation and aspect changes further compromise the accuracy. Thus, a diameter based upon a single infrared observation is unlikely to have an uncertainty less than $10 \%$ regardless of the precision of the infrared measurement.

\section{The Largest Asteroids}

By 1900 Barnard had established that Ceres was the largest of the "big four" (i.e., the four brightest asteroids) followed by Pallas and Vesta (which, to within one standard deviation, he found to be the same size). He found that Juno, at about one-fourth the size of Ceres, was the smallest of the big four. Compared with the best current values for the diameters of these four asteroids Barnard's sizes are systematically small by about $20 \%$; the relative sizes he determined are correct to within $12 \%$.

Using published photometry together with his diameter determinations Barnard derived albedos and noted that the albedo of Vesta was about four times greater than that of Ceres. (The best current estimate of the Vesta/Ceres albedo ratio is 3.7.)

Table I summarizes diameter determinations for the four brightest asteroids published between 1900 and 1993. Note that the radiometric diameters published by Allen (197.1) and TRIAD (Morrison and Zellner, 197.9) are not directly comparable to one another or to the those from the IRAS Minor Planet Survey (IMPS) due to changes in thermal models and calibrations of infrared standard stars. TRIAD diameters are about $17 \pm 3 \%$ larger with respect to IMPS results for the three largest asteroids. If the TRIAD polarimetric albedos are used together with the absolute magnitudes on the $H, G$ system (rather than on the $V(1,0)$ system) the polarimetric diameters increase by about $15 \%$ compared to the TRIAD values given below.

The diameters given in Table I are formally "mean diameters" only in the case of the Speckle and Occultation results. The others are approximations to mean diameters. The more measurements there are, averaged over both rotational phase and aspect angle, the closer to the mean diameter they will be. Because the four asteroids given in Table I are only slightly nonspherical the uncertainties in their diameters are dominated by random and systematic effects specific to the different techniques used to measure them. For example, before 197.9 it is clear that the results are greatly influenced by systematic effects and the quoted uncertainties are, in most cases, seriously underestimated. With the exception of Vesta (observed only once), the IMPS results, although all obtained at essentially a single aspect angle, sample between six and eight different rotational phases. IMPS diameters reported here and for Ceres, Pallas, and Juno and actual mean diameters, had 
sufficient observations been made to sample them, would be well within the quoted uncertainties. Note, however, that even had such observations been available the systematic differences, discussed in section 8 , below, would remain.

Diameter estimates published over the past 90 years for the three largest asteroids have been within about $1.5 \sigma$ of the actual mean diameters determined from observations of stellar occultations by these asteroids. The real improvement has been in the confidence in the quoted diameters. Prior to the mid-1980's the absolute uncertainties were about ten percent whereas today, thanks to the application of the stellar occultation technique, the uncertainty in the mean diameters of these asteroids is about $1 \%$.

TABLE I

A Summary of Diameter Determinations for the Four Brightest Asteroids (km).

\begin{tabular}{|c|c|c|c|c|c|c|c|}
\hline $\begin{array}{l}\text { Minor } \\
\text { Planet }\end{array}$ & $\begin{array}{c}\text { Barnard } \\
\text { (1900) } \\
\text { Filar } \\
\text { Microm. }\end{array}$ & $\begin{array}{c}\text { Allen } \\
\text { (1971) } \\
\text { Radiometry }\end{array}$ & $\begin{array}{l}\text { TRIAD } \\
\text { (1979) } \\
\text { Polarimetry }\end{array}$ & $\begin{array}{l}\text { TRIAD } \\
\text { (1979) } \\
\text { Rediometry }\end{array}$ & $\begin{array}{c}\text { Drum- } \\
\text { mond and } \\
\text { Hege } \\
\text { (1989) } \\
\text { Speckle }\end{array}$ & $\begin{array}{l}\text { Occul- } \\
\text { tation }\end{array}$ & $\begin{array}{c}\text { IMPS } \\
\text { (1993) } \\
\text { Rediometry }\end{array}$ \\
\hline Ceres & $770 \pm 90$ & $1160 \pm 80$ & $1016 \pm 100$ & $1014 \pm 100$ & & $933 \pm 5$ & $848 \pm 40$ \\
\hline Pallas & $490 \pm 120$ & & $692 \pm 70$ & $589 \pm 60$ & $503 \pm 33$ & $533 \pm 6$ & $498 \pm 38$ \\
\hline Juno & $195 \pm 25$ & $290 \pm 20$ & $252 \pm 25$ & $247 \pm 25$ & & $267 \pm 5$ & $234 \pm 22$ \\
\hline Vesta & $385 \pm 50$ & $570 \pm 10$ & $579 \pm 60$ & $530 \pm 50$ & $520 \pm 25$ & $520 \pm 10$ & $468 \pm 54$ \\
\hline
\end{tabular}

Notes to Table I.

1. Uncertainties are one standard deviation of Barnard's mean disk diameters at $1 \mathrm{AU}$ converted to $\mathrm{km}$. (All of Barnard's published diameters were in units of "english miles".)

2. Ceres - Millis et al. (1987); Pallas - Dunham et al. (1990); Juno - Millis et al. (1981); Vesta - Dunham (1991) [Uncertainty is from Dunham, personal communication.]

3. Uncertainties given here are twice the uncertainty in the precision tabulated in The IRAS Minor Planet Survey (1992).

The IRAS-derived radiometric diameters, although calibrated using the occultation diameters for Ceres and Pallas are systematically low relative to the occultation diameters for reasons discussed in the Summary section below. 
The vast majority of measured asteroid diameters are from radiometric observations. This has been the case since the late 1970's; TRIAD (Morrison and Zellner, 1979) lists polarimetric diameters for 28 asteroids and radiometric diameters for 192. Since then diameters from the other techniques discussed above have become available for about 25 asteroids. When available, quality A occultation diameters (as defined by Millis and Dunham, 1989) are generally the most reliable. Radiometric diameters, however, remain the most common due to the results from the IRAS survey which increased the number of asteroids with radiometric diameters to over 2,000 .

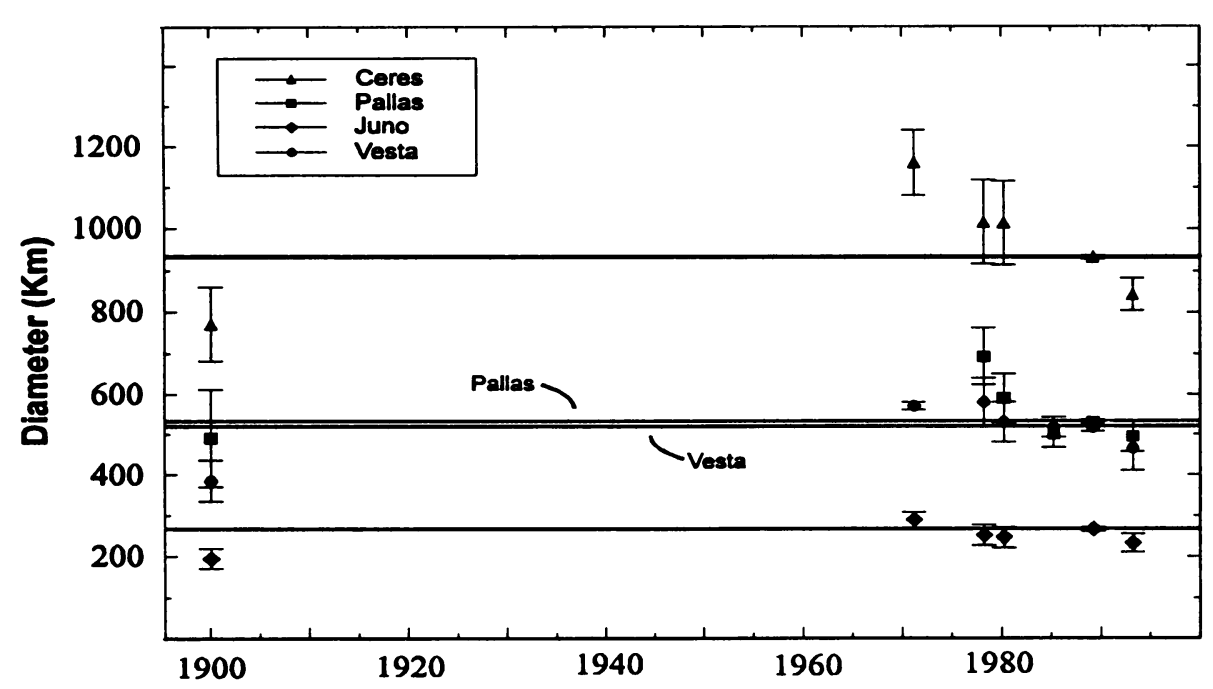

Fig. 1. Published diameters for the "big four" as a function of time. Horizontal lines indicate the best diameter estimates - all from stellar occultations. The techniques from which the diameters shown were obtained are, in chronological order, Filar Micrometry (Barnard, 1900), Infrared Radiometry (Allen, 1971), Polorimetry (Morrison and Zellner, 1979), Infrared Radiometry (Morrison and Zellner, 1979), Speckle (Drummond and Hege, 1989), Occultation (see note to Table I for references), and Infrared Radiometry (the IRAS Minor Planet Survey)

The first release of IRAS results (The IRAS Asteroid and Comet Survey, 1986) contained diameters for just under 1,800 asteroids. Many useful scientific results were obtained with this version. For example, it led to new estimates of the sizefrequency distributions of asteroids, an improvement in the standard asteroid thermal model, and the discovery of new taxonomic classes, to mention but a few. See Cellino et al. (1991), Matson et al. (1989), Tedesco et al. (1989a,b), and Veeder et al. (1989b) for further details. 
As good as the 1986 data products were, it was clear that (just as in the case of the IRAS Point Source Catalog) a better product could be produced using the experience gained during production of the first data set. In the case of the asteroids this meant searching the data stream using significantly more sets of (and more reliable) orbital elements, incorporating improved visual absolute magnitudes, accounting for the effects of flux overestimation (which affected about $40 \%$ of the observed asteroids), and better understanding the completeness versus reliability issue.

Thus between 1988 and 1992, under support provided by the Air Force Geophysics Laboratory, improved extraction and reduction routines were devised and implemented. Results for the approximately 2,000 asteroids with reliable IRAS observations are presented in an explanatory supplement entitled : The IRAS Minor Planet Survey (1992). There the history, processing, and analysis of these data are described. This document, together with machine-readable files of the final data products, have been deposited at the NSSDC under the name The IRAS Minor Planet Survey Database and Catalog supplanting the 1986 version IRAS Asteroid and Comet Database and Catalog. See the chapter in this book entitled : The IRAS Minor Planet Survey Data Base.

Due to the quantity and homogeneous nature of the IRAS Minor Planet Survey (IMPS) asteroid diameters and albedos the remainder of this paper will be devoted to an overview of the properties of that data set.

\section{IMPS Completeness}

The question of geometrical completeness of IRAS asteroid coverage is a complicated one. Because the survey was primarily concerned with inertially fixed sources, different parts of the sky were covered at different times, with the result that coverage was highly fragmented for any class of orbital elements (e.g., objects with the same elements except for the longitude of the ascending node). Here we use the expression "geometrical coverage" to mean that a given asteroid was swept by the IRAS field of view; a detection may or may not have occurred.

There are at least three geometrical factors that make the completeness of the IRAS survey different for the asteroids than for the inertially-fixed sources. The first two factors are independent of asteroid motion :

\section{A. The observed brightness of an asteroid depends on the IRAS viewing geometry.}

As the solar elongation angle of the IRAS scan changed, both the distance and phase angle (and hence the observed brightness) of an asteroid at a given ecliptic longitude varied. Therefore, the asteroid brightness distribution for a given ecliptic longitude was a function of the solar elongation angle(s) at which the observation scans were obtained.

The maximum variation occurred for the closest asteroids. For a ring of asteroids at $2 \mathrm{AU}$ from the sun, the geometrical distance effect causes a maximum variation in the flux of a factor of 3.1. The phase angle variation is too small to significantly affect the infrared flux. (The minimum phase angle is $25.7^{\circ}$ at $60^{\circ}$ or $120^{\circ}$ elongation, while the maximum phase angle is $30^{\circ}$ at $90^{\circ}$ elongation). 
In addition, the essentially Earth-centered IRAS viewing geometry caused an apparent variation in the density of asteroids with ecliptic longitude. The maximum observed density enhancement, caused by a variation of solar elongation scan angle from $60^{\circ}$ to $120^{\circ}$, is a factor of 1.8 .

$B$. The rate of coverage of the asteroid belt is significantly lower than the coverage of the inertial sky due to the Earth-centered observation frame

Although IRAS covered the inertially-fixed sky completely after six months, a gap remained in the asteroid coverage due to the motion of the Earth (see Fig. 2). Specifically, IRAS began the survey at ecliptic longitudes of approximately $60^{\circ}$ and $240^{\circ}$. Six months later, IRAS finished the first survey of the sky at those same longitudes. However, since the Earth was on the opposite side of the Sun from its original position, the part of the asteroid belt currently at ecliptic longitudes from approximately $62^{\circ}$ to $108^{\circ}$ (for asteroids at $2 \mathrm{AU}$ from the sun) remained unsurveyed. (Of course, since these scans went somewhere, this implies that other parts of the asteroid belt had a higher density of scans than the inertially-fixed sky.)

The third hours-confirming survey of the sky began by backing up to almost the maximum scan angle and resurveying the area of the sky just previously surveyed. When $60^{\circ}$ longitude was reached again, those asteroids were being seen for the first time, even though inertial sources were being resurveyed. Thus, ecliptic longitudes from $\sim 62^{\circ}$ to $\sim 108^{\circ}$ contain two, independent, surveys of different asteroids.

\section{Asteroidal motion}

Asteroidal motion causes several different effects. The major effect for asteroids with prograde orbits is to slightly lower the rate of coverage for those objects. For example, as mentioned above, without asteroid motion, for asteroids at $2 \mathrm{AU}$ from the sun, ecliptic longitudes from $\sim 62^{\circ}$ to $\sim 108^{\circ}$ were unsurveyed at the end of the first survey of the sky. Asteroidal motion amounts to about 20 degrees during the time it takes IRAS to observe that area, and hence IRAS must observe until longitude $130^{\circ}$ to entirely survey those asteroids.

In fact, ignoring the two major five degree gaps of the inertial coverage, IRAS was able to completely survey the asteroid belt except for prograde-orbiting asteroids having circular orbits with radii less than about 2.7 AU. Asteroids in such orbits moved too fast for IRAS to completely catch up to them.

A second effect due to asteroidal motion is caused by the short-term variations in the IRAS coverage. The IRAS survey used only about $60 \%$ of the IRAS observation time, with most of the rest of the time devoted to pointed observations. In addition, the survey was suspended due to the presence of the moon in a particular hemisphere for about 3 days per month. Because the IRAS survey was designed to ensure complete coverage of the inertial sky, these effects were compensated in carrying out the survey. However, because of Earth motion and asteroidal motion during those short-term hiatuses, there are significant local variations in coverage of the asteroids.

In order to obtain some feeling for the latter two effects, we ran a simulation using the actual survey observations and a set of hypothetical tracer asteroids. 


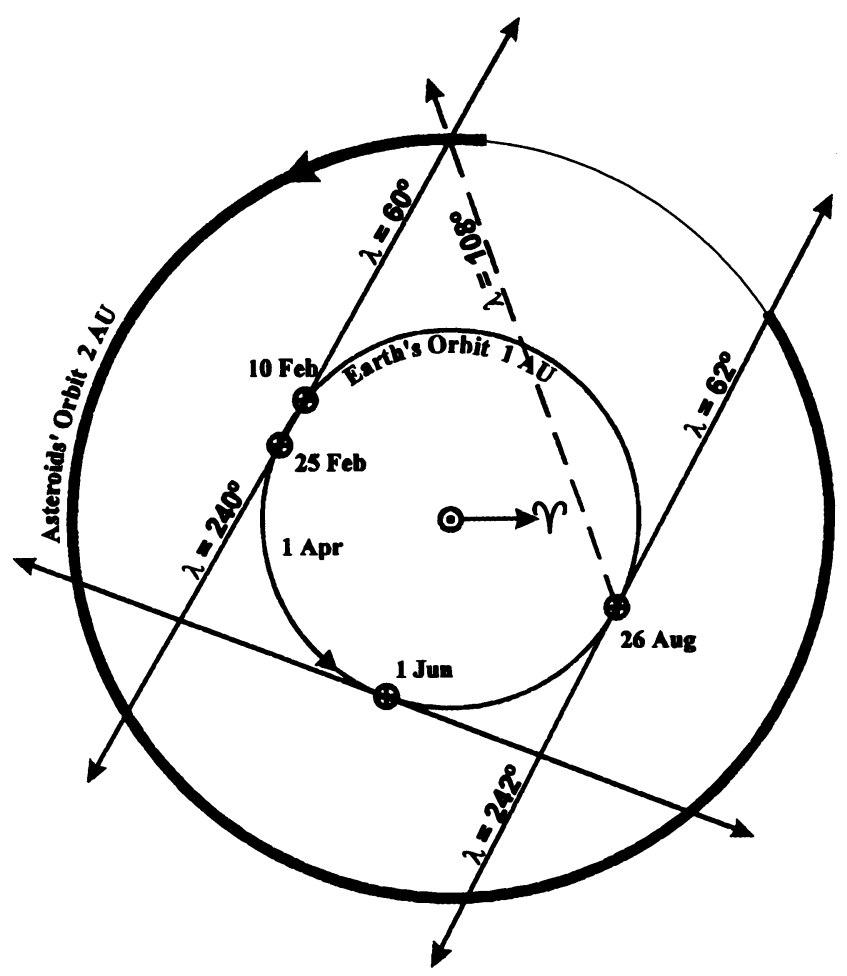

Fig. 2. Geometrical coverage of a ring at $2 \mathrm{AU}$ by the first six months of the IRAS mission. IRAS began the survey at ecliptic longitudes of $\sim 60^{\circ}$ and $240^{\circ}$. Approximately six months later, IRAS finished the first survey of the sky at about those same longitudes. However, since the Earth was then on the opposite side of the Sun from its position at the start of the mission, the part of the asteroid belt now at ecliptic longitudes from approximately $62^{\circ}$ to $108^{\circ}$ remained unsurveyed.

These tracer asteroids had circular orbits with zero inclinations. Ten evenly-spaced rings with semimajor axes from 2.0 to $5.5 \mathrm{AU}$ were populated with a total of 3,000 asteroids. These "asteroids" were assigned longitudes of the ascending node such that the linear density along the rings was constant. For each IRAS scan, all asteroids within 15 arcminutes of the IRAS boresight were counted as observed and the number of coverages was computed for each asteroid.

For this class of orbital elements, more than $94 \%$ of the tracers were observed at least once. The inner ring is the least complete because the higher rate of mo- 
tion allows tracers which lagged the coverage early to avoid being "lapped". The completeness varied over semimajor axis as shown in Table II.

Note that, except for the innermost orbits where the asteroidal motion prevented a full survey, the completeness for this set of asteroids is actually higher than that of the inertial survey! This is a direct result of the "slower" asteroidal-survey; the five degree gaps in the inertial sky are not quite as big for the asteroids. However, note that retrograding asteroids can (and a few near-Earth asteroids actually did) easily skip between IRAS scans, and hence the values given in Table II apply only to asteroids with elements similar to those assumed in this simulation.

TABLE II

Completeness Versus Semimajor Axis Ring for the Simulated Asteroids

\begin{tabular}{|c|c|}
\hline $\begin{array}{c}\text { Semimajor Axis } \\
(\mathrm{AU})\end{array}$ & Completeness \\
\hline $2.0-3.0$ & 0.944 \\
\hline $3.0-4.0$ & 0.988 \\
\hline $4.0-5.0$ & 0.983 \\
\hline $5.0-5.5$ & 0.979 \\
\hline Infinity & 0.973 \\
\hline
\end{tabular}

Finally, Table III shows detailed results of the number of times a simulated asteroid from a 2,000 asteroid per ring simulation was seen. Note that the mean coverage must always be the same within each ring. The "slower" coverage of the asteroids causes there to be many more asteroids observed a large number of times than for inertial sources.

TABLE III

Number of Times Simulated Asteroids Were Observed (2,000 in each ring)

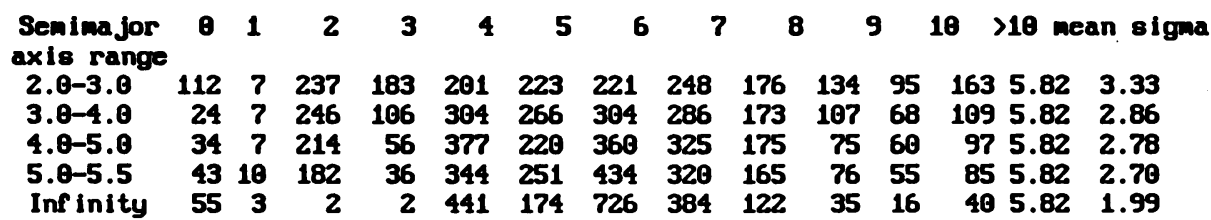




\section{Reliability}

There were 38,281 predicted asteroid crossings of the IRAS focal plane while it was in survey mode. Of these predicted sightings, 1,123 (a total of 232 different asteroids) had predicted $25 \mu \mathrm{m}$ flux densities that never exceeded $0.140 \mathrm{Jy}$ (the $1 \sigma$ limit).

Table IV summarizes the results of a test to assess the reliability of sources accepted as asteroids : 155 of the 1,123 potential sightings, for 31 of the 232 different asteroids, generated an association, i.e., a source with a $25 \mu \mathrm{m}$ flux density having an SNR $>3$ was found within the association ellipse for the putative asteroid. This implies that the false association rate is about $14 \%$. Note that all 155 of these false sightings were rejected by the IMPS data processing system; none were used in producing an accepted IMPS albedo and diameter. This further validates the reliability of the IMPS acceptance criteria. Furthermore, there is no significant difference in the false association rate between numbered and two or more opposition unnumbered asteroids. This is to be expected since these 232 asteroids are essentially a set of random positions near the ecliptic.

TABLE IV

Summary of $<1 \sigma$ Flux Limit Association Test

\begin{tabular}{||c|c|c|c|c||}
\hline ID Type & $\begin{array}{c}\text { Number of } \\
\text { Predicted } \\
\text { Sightings }\end{array}$ & $\begin{array}{c}\text { Number of } \\
\text { Predicted } \\
\text { Asteroids }\end{array}$ & $\begin{array}{c}\text { Number (\%) } \\
\text { of } \\
\text { Predictions } \\
\text { Associated }\end{array}$ & $\begin{array}{c}\text { Number (\%) } \\
\text { of Asteroids } \\
\text { Associated }\end{array}$ \\
\hline 1 & 140 & 26 & $24(17 \%)$ & $6(23 \%)$ \\
\hline 2 & 983 & 206 & $131(13 \%)$ & $25(11 \%)$ \\
\hline Totals: & 1,123 & 232 & $155(14 \%)$ & $31(13 \%)$ \\
\hline
\end{tabular}

\section{IMPS Vs. ADAS}

This section summarizes the major differences between ADAS (documented in IRAS Asteroid and Comet Survey, 1986) and the IMPS processing described in The IRAS Minor Planet Survey, 1992. There was one major difference in the association algorithm and two in the algorithms that computed the final albedos and diameters. These are discussed in detail below. The ground-based data sets used differed as well. ADAS processed asteroids through number 3318 plus 135 asteroids with two-or-more-opposition orbits while for IMPS these numbers were 4679 and 2,632 , respectively. ADAS used asteroid absolute magnitudes and slope parameters on the 1985 IAU system, while IMPS used the 1991 system. 


\section{1. THE ASSOCIATION ALGORITHM}

The ADAS association algorithm used a one-sigma positional uncertainty attributed to asteroids that was a function of the reliability of the orbital elements. In the course of the IMPS processing it was realized that the larger acceptance area used for asteroids with less-reliable orbital elements led to many spurious associations. This is especially true for faint asteroids. In IMPS a single one-sigma asteroid positional uncertainty was adopted. This single value (ten arcseconds) was, in effect, root-sum-squared with the IRAS Convolved Gaussian-Uniform positional uncertainty. This change virtually eliminated spurious associations for faint asteroids.

\section{2. THE FLUX OVERESTIMATION CORRECTION}

There is a systematic error associated with the measured flux densities near the noise limit of the detectors which can increase the reported value by as much as a factor of two compared with that of the true value. Weak sources were often detected when positive noise excursions pushed the source signal above the $3 \sigma$ SNR cutoff imposed by the IRAS processing. Negative excursions dropped the signal below the SNR cutoff and so were not detected. Thus, the flux densities of weak sources were systematically overestimated. This overestimation of the flux for low-SNR asteroid detections results in an overestimation of their diameters and an underestimation of their albedos.

Two correction methods have been used to correct this effect for inertial sources. Each of these methods assumes that the source is non-variable and the noise Gaussian. Unfortunately, (as shown by J. Fowler in Tedesco et al., 1992) each of the methods have an error in their derivations. Even were this not the case, without at least a priori knowledge of the luminosity function, these methods cannot be used to derive flux overestimation corrections.

Besides the above, the following are true of asteroidal sources but not of inertial sources.

1) Asteroids are intrinsically variable.

2) Asteroids move, thus increasing their "variability" through confusion.

3) About one-third of the accepted asteroids have fewer than three sightings; all inertial sources accepted into the PSC were sighted at least three times.

4) Over $95 \%$ of the accepted asteroid sightings are within $20^{\circ}$ of the ecliptic plane and hence were observed through the emission from the zodiacal cloud; the vast majority of inertial sources are outside this band and therefore were observed against a less noisy background.

The SNR correction method is rendered less certain by points 1,2 , and 3 , while point 4 implies that the SNR-based correction factors, as published by Cohen et al. (1987), are not applicable to asteroidal sources. For these reasons neither of these methods, even if they were valid, could be applied to asteroidal sources.

Thirty-nine percent of the accepted IRAS $25 \mu \mathrm{m}$ asteroid sightings, and $47 \%$ of the $12 \mu \mathrm{m}$ and $60 \mu \mathrm{m}$ sightings, are "weak sources", i.e., have SNR $<10$. IRAS asteroid diameters derived from "weak sources" are systematically large by an average of $\sim 33 \%$ with respect to ground-based observations. In our judgment, this effect was too large to ignore. We therefore decided to use the method originally proposed by Tedesco and Gradie (1988), i.e., to use the results from ground-based IRTF 
observations to derive a statistical correction for those IRAS asteroids affected by the flux-overestimation problem.

The upper portion of Fig. 3 shows a plot of the IRAS SNR versus the ratio of the $25 \mu \mathrm{m}$ flux density derived from ground-based (IRTF) observations to that reported by IRAS. The sample used consists of 801 accepted IRAS sightings for which high-quality albedos and diameters are available from IRTF observations by Gradie and Tedesco (1988). The albedo and diameter derived from each IRTF observation were used to predict the $25 \mu \mathrm{m}$ flux density at the time of each IRAS sighting of that asteroid. The data were grouped into logarithmic bins 0.1 unit wide and the mean IRTF/IRAS flux density ratio and its standard deviation were then computed for each bin.

The quantities plotted were chosen to facilitate comparison between the flux overestimation correction method derived here and that used in correcting the IRAS Point Source Catalog Ver. 1 flux densities (cf., IRAS Explanatory Supplement, 1988 : Fig. XII.A.2, p. XII-6). Note that for SNR $>10$ the agreement is good but that below this value the IRAS fluxes are systematically higher than those predicted on the basis of the IRTF observations.

Because the IRTF data do not have an SNR-imposed cutoff the fluxes derived from them do not have an SNR-related systematic error. We therefore derived a linear correction factor as a function of SNR based upon the departure between the IRTF and IRAS fluxes. The correction factor was 0.725 at $\mathrm{SNR}=3.0$ and 1.0 at $\mathrm{SNR}=10.0$.

The correction was applied to all detected fluxes with signal-to-noise ratios (SNR) between 3.0 and 10.0. The results of applying this correction factor to the IRAS measurements in the sample used to derive the correction factors is shown in the lower portion of Fig. 3.

To within the limits of measurement the correction factor at $12 \mu \mathrm{m}$ is the same as that for $25 \mu \mathrm{m}$. Because no ground-based measurements are available at $60 \mu \mathrm{m}$ we are unable to derive a correction for observations made in this band and simply assume that it is the same as that for the shorter bands. Thus, we subsequently applied this single correction factor to all IRAS asteroid detections with SNR < 10 before using them to derive IRAS albedos and diameters. This was done before iteratively computing the albedo and diameter. The flux uncertainty for corrected bands was increased by root-sum-squaring it with the flux correction. The flux overestimation correction factor, if any, applied to each accepted sighting is part of the IMPS data set.

\section{Band-to-Band Albedo Discrepancies}

Albedos derived from 25 or $60 \mu \mathrm{m}$ IRAS fluxes are systematically about $10 \%$ higher than those derived from $12 \mu \mathrm{m}$ IRAS fluxes. This could, for example, be caused by an error in the IRAS flux calibration or an invalid assumption in the asteroid thermal model (perhaps the thermal emissivity and/or beaming parameter are wavelength-dependent). Regardless of the cause, the effect is to introduce erroneous differences in the albedos derived from $12 \mu \mathrm{m}$ detections and those derived from 25 and $60 \mu \mathrm{m}$ detections. 

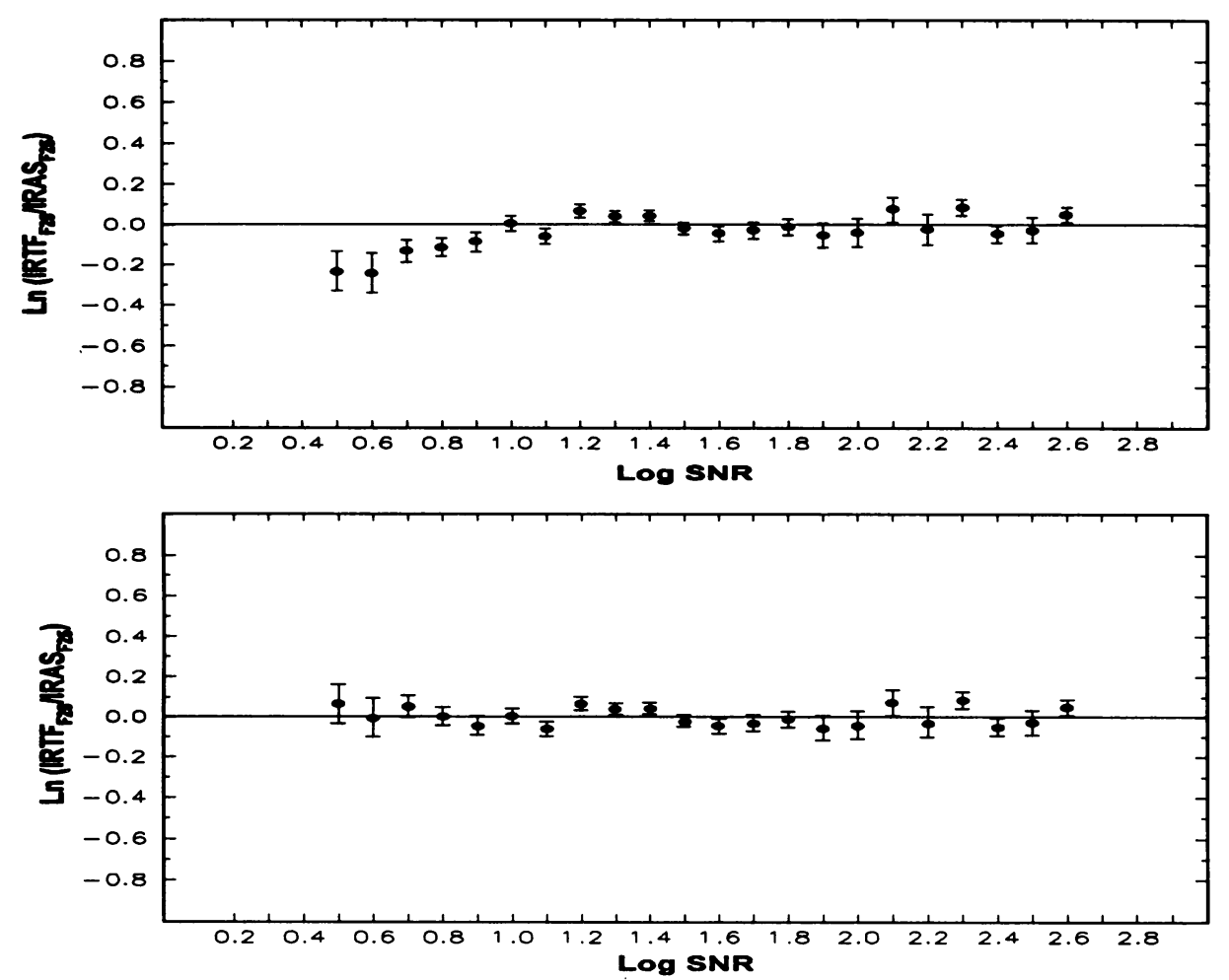

Fig. 3. Common logarithm of the signal-to-noise versus the natural logarithm of the mean ratio of $25 \mu \mathrm{m}$ flux densities from IRTF and IRAS observations per SNR bin. The uncorrected data are presented in the upper figure and the corrected in the lower.

To eliminate this discrepancy we decided to adopt the albedos derived from either $12 \mu \mathrm{m}$ or $25 \mu \mathrm{m}$ detections as being "correct". To aid in this choice the diameters based on $12 \mu \mathrm{m}$-only and $25 \mu \mathrm{m}$-only data were compared with 13 asteroids with high-quality diameters obtained from stellar occultations. The mean difference between the occultation-derived diameters and the $12 \mu \mathrm{m}$-derived diameters was $6.5 \%$ while that for the $25 \mu \mathrm{m}$-derived diameters was $7.0 \%$. We therefore adopted the $12 \mu \mathrm{m}$-derived diameters as being "correct". Subsequently, we learned that this choice is consistent with the IRAS calibration error discussed in Cohen et al., (1992). Hence, a correction factor of 1.12 was applied to the albedos (and therefore, indirectly, to the diameters computed from them) to all albedos derived from $25 \mu \mathrm{m}$ and $60 \mu \mathrm{m}$ fluxes. Following this correction the mean difference between the occultation-derived diameters and the $25 \mu \mathrm{m}$-derived diameters was reduced to $6.7 \%$

Figure 4 shows plots of the differences between albedos derived from $12 \mu \mathrm{m}$ fluxes and those determined from 25 and $60 \mu \mathrm{m}$ fluxes. 

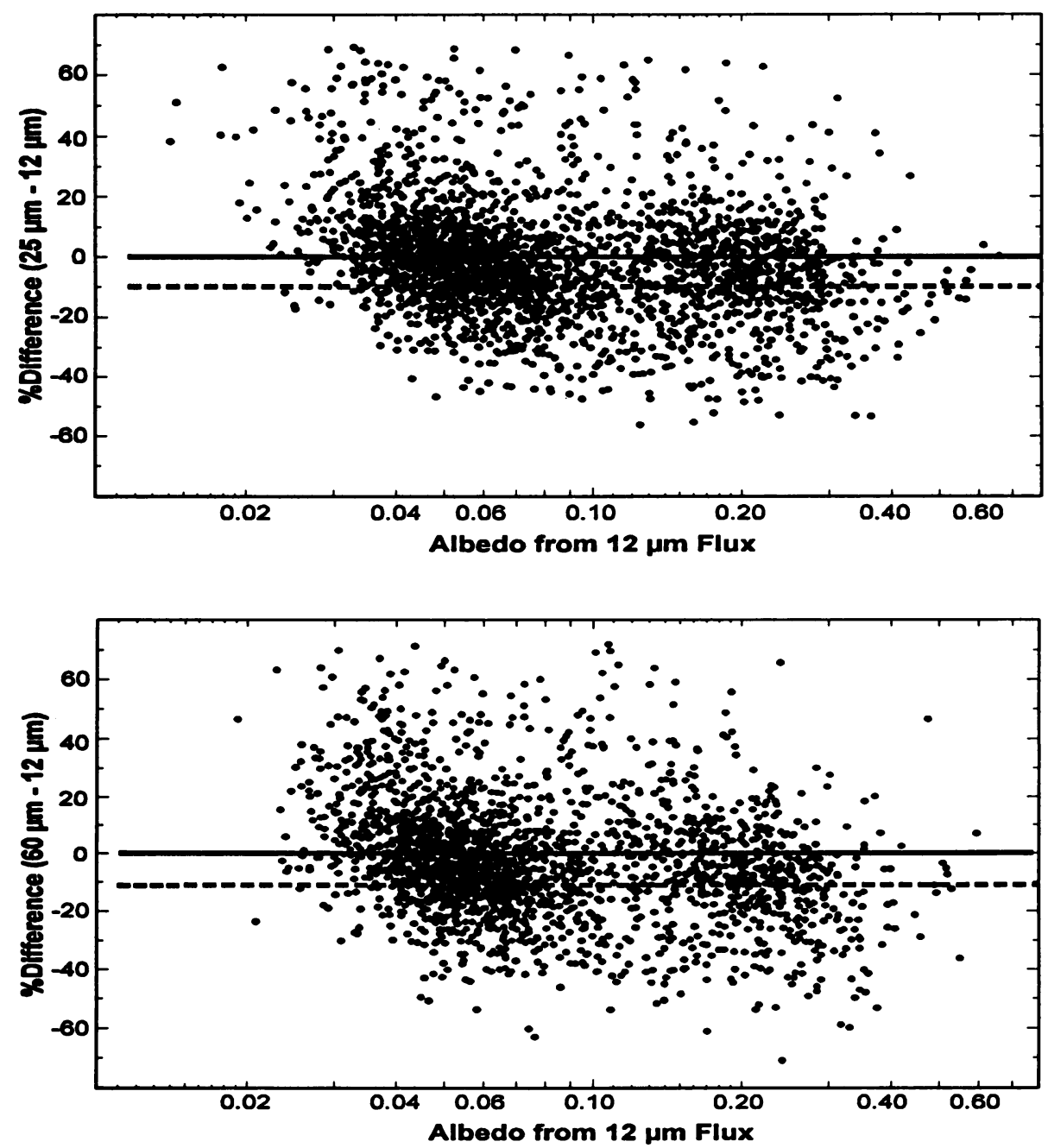

Fig. 4. Comparison of model albedos obtained from data at 12,25 , and $60 \mu \mathrm{m}$. The 12 vs. $25 \mu \mathrm{m}$ results are displayed at the top and the 12 vs. $60 \mu \mathrm{m}$ results at the bottom. In each case the uncorrected mean difference is indicated by the dashed line and the corrected difference by the solid line. Due to the large size of the graphics file ( $\sim 3 \mathrm{Mb})$ only every other data point is plotted in these figures.

Table V presents statistics on the $12 / 25 \mu \mathrm{m}$ and $12 / 60 \mu \mathrm{m}$ distributions before and after application of the band-to-band correction. 
TABLE V

Sample Statistics for Corrected Vs. Uncorrected $12 \mu \mathrm{m} / 25 \mu \mathrm{m}$ and $12 \mu \mathrm{m} / 60 \mu \mathrm{m}$ Albedos

\begin{tabular}{||c|c|c|c|c|c|c|c|}
\hline Bands & N & \multicolumn{2}{|c|}{ Mean \% Diff } & \multicolumn{2}{|c|}{ Sample } & \multicolumn{2}{c|}{$\%>20$} \\
\hline & & Before & After & Before & After & Before & After \\
\hline $12 / 25$ & 5,763 & -9.9 & +0.9 & 20.7 & 23.2 & 5.9 & 4.9 \\
\hline $12 / 60$ & 4,884 & -11.1 & -0.5 & 21.7 & 24.3 & 2.9 & 4.6 \\
\hline
\end{tabular}

The albedos and diameters given in the IMPS final data products have had both the flux-overestimation and band-to-band corrections applied. After applying these corrections the data were again examined for systematic band-to-band effects. It was found that the mean difference in albedos derived from $12 \mu \mathrm{m}$ flux densities and those derived from $25 \mu \mathrm{m}$ or $60 \mu \mathrm{m}$ flux densities is less than $1 \%$ for the entire sample.

\section{1. ADAS VERSUS IMPS SIGHTINGS OF NUMBERED ASTEROIDS}

Using orbital elements for asteroids numbered through 3318 ADAS processing found 1,790 asteroids with one or more accepted sightings whereas the IMPS processing produced 1,678 such asteroids. As discussed above, this difference is due to the more-stringent positional match requirement used in the IMPS processing. The total number of asteroids with accepted IMPS sightings is 1,890 due to the greater number of available orbital elements. IMPS also produced fewer rejected asteroid sightings (and more missed sightings) than ADAS, implying that the initial tagging by IMPS of an IRAS detection as a "potential sighting" is more reliable than the association method used in the ADAS processing. See Fig. 5.

\section{Apparent Albedo Distribution}

Figure 6 shows the apparent albedo distribution of the asteroids observed by IRAS. The data are shown separately for the larger and smaller asteroids with $44 \mathrm{~km}$ being chosen as the dividing diameter. Note that the distribution for the larger asteroids is clearly bimodal with the dividing minimum near an albedo of 0.1 . The distribution for the smaller asteroids is certainly different. Part of this difference can be attributed to differences in the selection effects for small and large numbered asteroids. The larger numbered asteroids contain a disproportionate number of higher albedo members. Not so easily explained is the difference in the proportion of intermediate albedo asteroids in the two samples : Only $5 \%$ of asteroids with D $>44 \mathrm{~km}$ have IMPS albedos between 0.09 and 0.14 whereas $20 \%$ of the smaller asteroids have IMPS albedos in this range. 


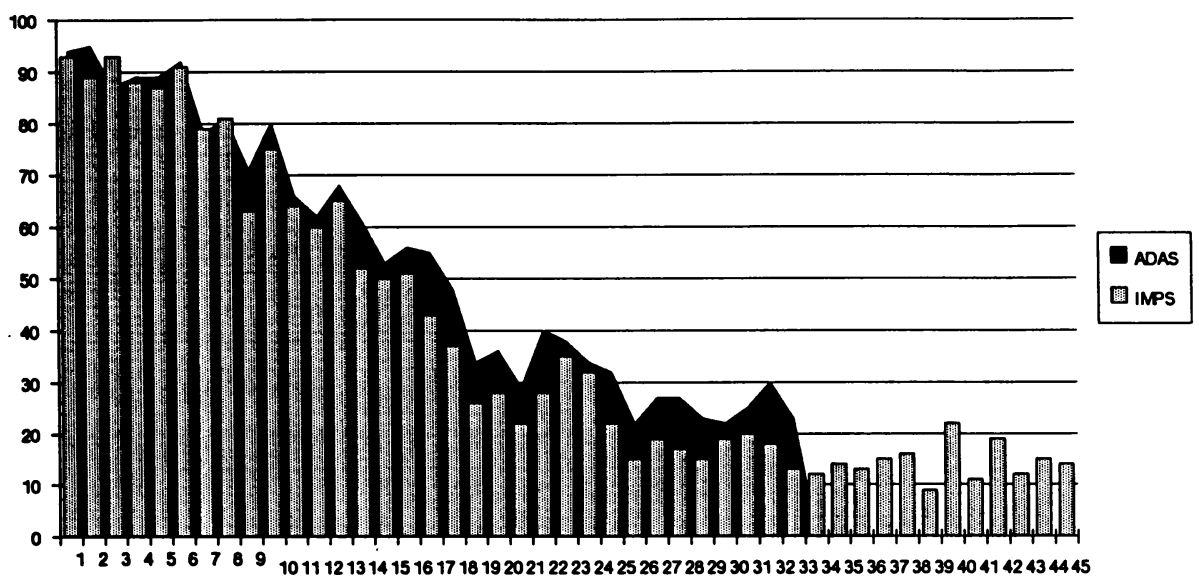

Fig. 5. Percent of numbered asteroids with accepted ADAS and IMPS observations. The area and bar chart peaks represent the actual number of asteroids, per 100 asteroid wide bin, with accepted ADAS and IMPS observations, respectively. Note that ADAS has more accepted asteroid observations in most bins, a fact attributable to the less-stringent positional match requirement compared with that used in the IMPS processing. ADAS processed 3,318 numbered asteroids and IMPS 4,679. Note that more than $10 \%$ of asteroids with numbers greater than 2500 yield reliable data.

\section{Summary}

IMPS diameters for the largest asteroids are systematically low with respect to occultation diameters (cf., Fig. 1). This difference is about $10 \%$ or $1 \sigma$ except for Ceres which differs by $9 \%$ or $2 \sigma$. The reason for this is that although, on average, the band-to-band correction improved the agreement in the albedos among results derived from $12 \mu \mathrm{m}$ fluxes and those derived from 25 and $60 \mu \mathrm{m}$ fluxes (from $10 \%$ to $<1 \%$ ) the agreement actually worsened for a few of the largest asteroids. In particular, the diameter obtained for Ceres from $12 \mu \mathrm{m}$ fluxes alone differs, following the band-to-band correction, by about $8 \%$ (which corresponds to $10 \sigma$ !) with respect to that derived from $25 \mu \mathrm{m}$ fluxes.

Apparently, the need for the band-to-band correction is due to the fact that Ceres was used to "calibrate" the standard thermal model by requiring results based upon observations at 10 and $20 \mu \mathrm{m}$ to simultaneously match the diameter obtained from the stellar occultation (Lebofsky et al., 1986a,b), a not unreasonable restriction. However, this requirement led to the difference seen in the IMPS-derived albedos and diameters for virtually all other asteroids. Thus the parameters in the standard 


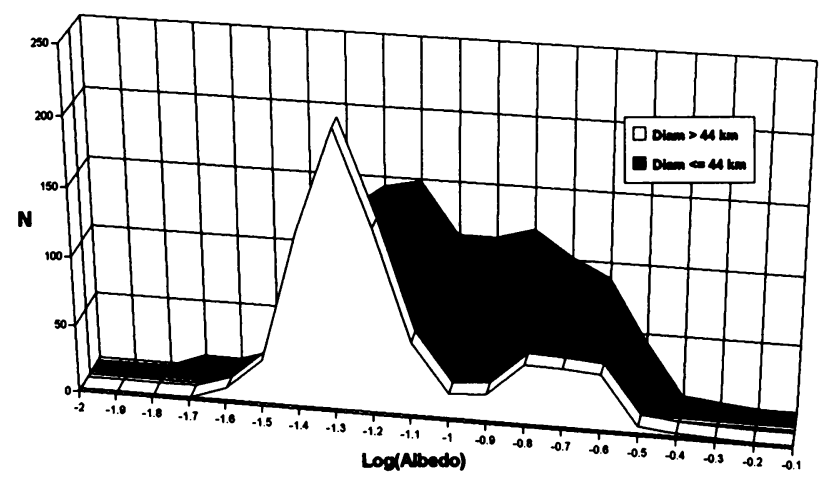

Fig. 6. IMPS albedo distribution for two asteroid size-groups.

thermal model for wavelengths $>10 \mu \mathrm{m}$ are correct for Ceres but not for the vast majority of smaller asteroids. Hence, the thermal properties of Ceres, and perhaps those of other asteroids with diameters $\gg 200 \mathrm{~km}$, differ significantly from those of smaller asteroids. For example, the thickness, mineralogy, and/or particle-size distribution in the regoliths of these largest asteroids may differ appreciably from those of smaller asteroids.

For intermediate-size asteroids, i.e., those with diameters greater than $100 \mathrm{~km}$ but less than $350 \mathrm{~km}$, the IMPS results appear accurate to better than $10 \%$. This is based upon a comparison of 13 IMPS asteroids for which there exist high-quality results from stellar occultations. The mean difference in the diameters from these two techniques is $7 \%$.

The albedo size-dependence seen in the IMPS data may be real and due to the lack of a mature dusty regolith on many small asteroids (Veeder,1991). This view is supported by results from ground-based observations as well. For example, Tedesco et al. (1990) computed the "10 $\mu$ m albedo" / $20 \mu \mathrm{m}$ albedo" ratio $\left(\mathrm{p}_{10} / \mathrm{p}_{20}\right)$ for 352 dual-wavelength observations obtained in the Gradie and Tedesco IRTF Asteroid Radiometry Survey (Gradie and Tedesco, 1988) and then performed linear leastsquares fits to this ratio versus the diameter, heliocentric distance at the time of observation, and the " $10 \mu \mathrm{m}$ albedo". They found that there is no correlation between the albedo ratio and the heliocentric distance or albedo but a strong correlation between the albedo ratio and the diameter. They concluded that the thermal properties of asteroids vary in a statistically significant way with size but not with albedo or distance. The sense of this variation is consistent with many smaller asteroids having surfaces with a larger rock/dust component (larger mean particle size) than the typical large asteroid.

If this is indeed the case, then radiometric observations of asteroids with diameters less than about $40 \mathrm{~km}$ cannot be used to derive accurate albedos or diameters. For asteroids below this size the standard thermal model can produce albedos which 
are too high by as much as a factor of two (cf., Veeder et al., 1989a). Thus applications such as derivations of size-frequency distributions or albedo-dependent taxonomic classifications may produce unreliable results if they include asteroids below this size.

To the extent that "the science is in the details" the IMPS data base is a significant improvement over the earlier ADAS product. Due to space limitations, and to avoid being overly pedantic, I have not attempted to contrast every difference between the two data bases. Suffice it to say that the major conclusions drawn from the ADAS data base are supported by IMPS but significant differences in the details do exist.

\section{Acknowledgements}

The work described herein was made possible through support provided by Dr. Stephan D. Price, Phillips Laboratory, Geophysical Directorate. I also thank Paul Noah of MRC for creating Fig. 2 and for sharing his knowledge on how IRAS surveyed the non-inertial sky. Finally, I hereby acknowledge the contributions by Tom Chester and John Fowler of IPAC to Chapter 8 of The IRAS Minor Planet Survey, 1992 from which portions of the present paper have been abstracted, and to Drs. R. Binzel and V. Zappalá for constructive criticisms on the penultimate version of this paper.

\section{References}

Allen, D.A. : 1971, "The method of determining infrared diameters". In Physical Studies of Minor Planets (T. Gehrels, Ed.), NASA SP-267, pp. 41-44.

Barnard, E.E. : 1894, "On the diameters of Ceres, Pallas, and Vesta". Astron. $\&$ Astrophys. 13, 341-344.

Barnard, E.E. : 1895, "Micrometrical determinations of the diameters of the minor plan ets Ceres (1), Pallas (2), Juno (3), and Vesta (4), made with the filar micrometer of the 36-inch equatorial of the Lick Observatory, and on the albedos of those planets". Monthly Not. Roy. Astron. Soc. 56, 55-63.

Barnard, E.E. : 1900a, "On the diameter of Ceres and Vesta". Monthly Not. Roy. Astron. Soc. 60, 261-263.

Barnard, E.E. : 1900b, "The diameter of the asteroid Juno (3) determined with the micrometer of the 40 -inch refractor of the Yerkes Observatory, with remarks on some of the other asteroids". Monthly Not. Roy. Astron. Soc. 61, 68-69.

Cellino, A., Zappala, V., and Farinella, P.: 1991, "The size distribution of main-belt asteroids from IRAS data". Monthly Not. Roy. Astron. Soc. 253, 561-574.

Cohen, M., Schwartz, D.E., Chokshi, A., and Walker, R.G. : 1987, "IRAS colors of normal stars". Astron. J. 93, 1199-1219.

Dollfus, A. : 1971, "Diameter measurements of asteroids". In Physical Studies of Minor Planets (T. Gehrels, Ed.), NASA SP-267, pp. 25-32.

Dollfus, A., Wolff, M., Geake, J.E., Lupishko, D.F., and Dougherty, L.M. : 1989, "Photopolarimetry of asteroids". In Asteroids II (R.P. Binzel, T. Gehrels, and M.S. Matthews, Eds.), University of Arizona Press, Tucson, pp. 594-616.

Drummond, J.D. and Hege, E.K. : 1989, "Speckle interferometry of asteroids". Ibid, pp. 171-191.

Dunham, D.W. : 1991, "Asteroid news". Occultation Newsletter 5, 93. 
Dunham, D.W. and 45 others : 1990, "The size and shape, of (2) Pallas from the 1983 occultation of 1 Vulpeculae". Astron. J. 99, 1636-1662.

Gradie, J. and Tedesco, E.F. : 1988, "Albedos and diameters for 350 asteroids from the IRTF $10 \mu \mathrm{m}$ and $20 \mu \mathrm{m}$ radiometry survey". Bull. Amer. Astron. Soc. 20, 866.

The IRAS Minor Planet Survey, 1992, edited by E.F. Tedesco. Phillips Laboratory Technical Report No. PL-TR-92-2049. Hanscom Air Force Base, MA.

Infrared Astronomical Satellite Asteroid and Comet Survey : Preprint Version No. 1, 1986, edited by Matson, D.L., JPL Internal Document No. D-3698. (Jet Propulsion Laboratory, Pasadena)

IRAS Catalogs and Atlases, Volume 1, Explanatory Supplement, 1988, edited by Beichman, C.A., Neugebauer, G., Habing, H.J., Clegg, P.E. and Chester, T.J. (NASA Publication No. RP-1190, Washington D.C. : U.S. Government Printing Office).

KenKnight, C.E., Rosenberg, D.L., and Wehner, G.K. : 1967. "Parameters of the optical properties of the lunar surface powder in relation to solar wind bombardment". J. Geophys. Res. 72, 3105-3117.

Lebofsky, L.A. and Spencer, J.R. : 1989. "Radiometry and thermal modeling of asteroids". In Asteroids II (R.P. Binzel, T. Gehrels, and M.S. Matthews, Eds.), University of Arizona Press, Tucson, pp. 128-147.

Lebofsky, L.A., Matson, D.L., Veeder, G.J., and Tedesco, E.F. : 1986a. "The IRAS asteroid thermal model". In Infrared Astronomical Satellite Asteroid and Comet Survey, D.L. Matson, ed. (JPL D-3698, Jet Propulsion Laboratory, Pasadena), pp. 7-1 to 7-18.

Lebofsky, L.A., Sykes, M.V., Tedesco, E.F., Veeder, G.J., Matson, D.L., Brown, R.H., Gradie, J.C., Feierberg, M.A. and Rudy, R.J. : 1986b. "A refined "standard" thermal model for asteroids based on observations of 1 Ceres and 2 Pallas". Icarus 68, 239-251.

Matson, D.L., Veeder, G.J., Tedesco, E.F., and Lebofsky, L.A. : 1989. "The IRAS Asteroid and Comet Survey". In Asteroids II, R. Binzel, T. Gehrels and M. Matthews, eds. (University of Arizona Press, Tucson), pp. 269-281.

Millis, R.L. and Dunham, D.W. : 1989. "Precise measurement of asteroid sizes and shapes from occultations". In Asteroids II (R.P. Binzel, T. Gehrels, and M.S. Matthews, Eds.), University of Arizona Press, Tucson, pp. 148-170.

Millis, R.L. and 37 others : 1981. "The diameter of Juno from its occultation of AG $+0^{\circ}$ 1022". Astron. J. 86, 306-313.

Millis, R.L. and 41 others : 1987, "The size, shape, density, and albedo of Ceres from its occultation of BD $+8^{\circ} 471$ ". Icarus 72, 507-518.

Morrison, D. and Zellner, B. : 1979, "Polarimetry and radiometry of the asteroids". In Asteroids II (R.P. Binzel, T. Gehrels, and M.S. Matthews, Eds.), University of Arizona Press, Tucson, pp. 1090-1097.

Ostro, S.J.: 1989, "Radar observations of asteroids". In Asteroids II (R.P. Binzel, T. Gehrels, and M.S. Matthews, Eds.), University of Arizona Press, Tucson, pp. 192212.

Tedesco, E.F. : 1989a, "Introduction to the Asteroids II data base". In Asteroids II, (R. Binzel, T. Gehrels and M. Matthews, Eds.) pp. 997-1001, University of Arizona Press, Tucson.

Tedesco, E.F. : 1989b, "Asteroid magnitudes, UBV colors and IRAS albedos and diameters". In Asteroids II, (R. Binzel, T. Gehrels and M. Matthews, Eds.) pp. 1090-1138, University of Arizona Press, Tucson.

Tedesco, E.F. and Gradie, J.C. : 1988, "IRAS Vs. IRTF asteroid radiometric albedos". Bull. Amer. Astron. Soc. 20, 865.

Tedesco, E.F., Gradie, J.C., and Lebofsky, L.A. : 1990, "Size dependence of radiometric diameters and albedos". Bull. Amer. Astron. Soc. $22,1113$.

Tedesco, E.F., Fowler, J.W., and Chester, T.J. : 1992, "Summary". In Infrared Astronomical Satellite Minor Planet Survey Catalog, 1992 (E.F. Tedesco, Ed.), pp. 127-148. Phillips Laboratory Technical Report No. PL-TR-92- 2049. Hanscom Air Force Base, MA. 
Tedesco, E.F., Matson, D.L. and Veeder, G.J. : 1989a, "Classification of IRAS asteroids". In Asteroids II, R. Binzel, T. Gehrels and M. Matthews, eds. (University of Arizona Press, Tucson), pp. 290-297.

Tedesco, E.F., Williams, J.G., Matson, D.L., Veeder, G.J., Gradie, J.C. and Lebofsky, L.A. : 1989b, "A three-parameter asteroid taxonomy". Astron. J. 07, 580-606.

Veeder, G.J. : 1991, "Evidence against dusty regoliths on small main belt asteroids". Bull. Amer. Astron. Soc. $28,1155$.

Veeder, G.J., Hanner, M.S., Matson, D.L., Tedesco, E.F., Lebofsky, L.A. and Tokunaga, A.T. : 1989a, "Radiometry of near-Earth asteroids". Astron. J. 97, 1211-1219.

Veeder, G.J., Tedesco, E.F., and Matson, D.L. : 1989b, "Asteroid results from the IRAS survey". In Asteroids II, R. Binzel, T. Gehrels and M. Matthews, eds. (University of Arizona Press, Tucson), pp. 282-289.

Zellner, B. Leake, M., Le Bertre, T., Duseaux, M., and Dollfus, A. : 1977a, "The asteroid albedo scale. I - Laboratory polarimetry of meteorites". Proc. Lunar Sci. Conf. 8, 1091-1100.

Zellner, B. Le Bertre, T., and Day, K. : 1977b, "The asteroid albedo scale. II - Laboratory polarimetry of dark carbon-bearing silicates". Proc. Lunar Sci. Conf. 8, 1111-1117. 